\title{
Comprehensive Sexuality Education (CSE) Curriculum in 10 East and Southern African Countries and HIV Prevalence among the Youth
}

\author{
Miracle Ayomikun Adesina ${ }^{1,2,3,4,5 *}$, Isaac I. Olufadewa ${ }^{1,6}$
}

\author{
${ }^{1}$ Slum and Rural Health Initiative Research with Purpose Academy, NIGERIA \\ ${ }^{2}$ Cephas Health Research Initiative Inc, Ibadan, NIGERIA \\ ${ }^{3}$ Mental and Oral Health Development Organization, Kebbi State, NIGERIA \\ ${ }^{4}$ Universal Care for Africa Foundation, St. Loius, USA \\ ${ }^{5}$ Department of Physiotherapy, University of Ibadan, Ibadan, NIGERIA \\ ${ }^{6}$ Pan African University of Life and Earth Sciences Institute, PAULESI, Ibadan, NIGERIA \\ *Corresponding Author: miracleadesina5@gmail.com
}

Citation: Adesina, M. A. and Olufadewa, I. I. (2020). Comprehensive Sexuality Education (CSE) Curriculum in 10 East and Southern African Countries and HIV Prevalence among the Youth. European Journal of Environment and Public Health, 4(1), em0035. https://doi.org/10.29333/ejeph/6009

\begin{tabular}{lll}
\hline ARTICLE INFO & ABSTRACT \\
\cline { 1 - 1 } Received: 26 Jul. 2019 & $\begin{array}{l}\text { Introduction: Young people easily fall prey to risky and dangerous practices such as drug abuse, unprotected } \\
\text { sexual intercourse etc. due to lack of knowledge about the implications of these practices. Comprehensive }\end{array}$ \\
Revised: 03 Sep. 2019 & $\begin{array}{l}\text { Sexuality Education (CSE) was developed to solve this problem by educating young minds so that they can avoid } \\
\text { risky sexual behaviours and live healthy lives. }\end{array}$ \\
Accepted: 04 Sep. 2019 &
\end{tabular}

Methods: The ten countries in East and Southern Africa selected were those whose CSE curriculum had been reviewed by the United Nations Educational, Scientific and Cultural Organization (UNESCO) and United Nations Population Fund (UNFPA). We developed a scoring protocol to be able to specifically group a curriculum into three categories (A, B and C) based on its weakness. The data for HIV was based on the UNAIDS estimation. Data was analyzed with the Microsoft Excel Version 2010.

Results: Sixty (60\%) percent of the countries involved had CSE curriculum with minor to moderate concerns, while the remaining $40 \%$ had moderate to serious gaps. Botswana and Lesotho had high prevalence of HIV among both young males and females. Majority of the countries with CSE curriculum having minor to moderate gaps had high prevalence of HIV among males within age 15-24 years. In the female category, one of the countries using CSE curriculum with moderate to serious gaps had an average HIV prevalence higher than the average of all the 10 countries involved in the study.

Conclusion: It is recommended that factors responsible for high HIV prevalence as identified in this study be addressed. This might help reduce the prevalence of HIV.

Keywords: sexuality, education, curriculum, HIV, East Africa, Southern Africa, SIMBIHealth

\section{INTRODUCTION}

It is well established that the young mind is very inquisitive and the young sometimes experiment to find out about some issues from a personal experience (Kidd and Hayden, 2015). Young people therefore easily fall prey to illicit, risky and dangerous practices which relates to drug use, unprotected sexual intercourse etc. (Kohli et al., 2018). Lack of knowledge about sexuality issues such as unsex safe among others may be why a lot of youngsters fall into this (Kyilleh, Tabong and Konlaan, 2018). Also, young persons need to make decisions as regards their sexual nd reproductive health however, research shows that majority of them lack adequate knowledge to make appropriate decisions (UNFPA, 2016). Educational and technological innovations such as SIMBIHealth (Sexuality Information, Mental and Behavioural Initiative for Health) (SIMBIHealth, 2019) and Comprehensive Sexuality Education (CSE) (Panchaud et al., 2018), among others were developed to solve the problem and educate the young minds.

Comprehensive sexuality education is a curriculum-based process of teaching and learning about the cognitive, emotional, physical, social and even the spiritual aspects of sexuality. It is delivered in both formal and non-formal 
settings (UNESCO and UNFPA, 2012). CSE is a scientifically accurate, incremental, age- and developmentally appropriate, curriculum based on a human rights approach. It is culturally relevant and context appropriate, transformative and able to develop the life skills needed to support healthy choices among young people while promoting gender equality (UNESCO and UNFPA, 2012).

Full implementation of comprehensive sexuality education curriculum in the Africa continent faces many challenges (Keogh et al., 2018). Some individuals and groups believe that CSE leads to early sexual initiation and deprives children of their innocence. Some parents object to sexuality education being taught in schools. Some state that CSE is a means of recruiting young people towards alternative lifestyles and it is inappropriate for young children (UNESCO and UNFPA, 2012).

This study aims to investigate the association between the prevalence of HIV in ten (10) countries in Eastern and Southern Africa and the strength or weakness of the CSE curriculum been used in those countries. The outcome of the study will serve as additional evidence and support for full implementation of CSE in Africa.

\section{METHODS}

The ten countries in East and Southern Africa selected were those whose comprehensive sexuality education curriculum had been reviewed by the United Nations Educational, Scientific and Cultural Organization (UNESCO) and United Nations Population Fund (UNFPA). The ten countries that were involved in this study are Botswana, Kenya, Lesotho, Malawi, Namibia, South Africa, Swaziland (Eswatini), Uganda, Zambia and Zimbabwe. The Ministry of Education staffs from all the ten countries along with other stakeholders were involved in this review. A scanning instrument was developed to determine if the countries curriculum meets the standard of CSE (UNESCO and UNFPA, 2012).

A scanning instrument was developed by the collaboration of the Population Council, UNESCO and UNFPA regional offices for ESA plus the relevant UNESCO and UNFPA headquarters offices. The scanning instrument was made to allow for documenting whether the important content was included. The instrument also contains various items on various issues such as age-appropriateness, behavioural goals the curriculum was aiming to promote, diversity and effectiveness of teaching methods along with sensitivity to gender and rights across topics (UNESCO and UNFPA, 2012).

The curriculums were reviewed following the standard CSE topics (6), topics and approach (3) and teaching approach. The findings were categorized based on the gaps in the curriculum in comparison to the Standard CSE. The four categories are (UNESCO and UNFPA, 2012).

1. No significant gap

2. Topics or areas that are missing or weak

3. Concerns that are minor to moderate

4. Concerns that are moderate to serious

We developed a scoring protocol to be able to specifically group a curriculum into three categories (A, B and C) based on weakness in it. Category 1, based on UNESCO and UNFPA finding was given a score of 1 , category 3 was assigned a score of 2 and category 4 finding was scored 3. No scoring was assigned to category 2 has it was interlinked with the others and scoring it would lead to tautology. Countries with higher scores have higher gaps or concerns in their CSE curriculum. A score of 12-20 was interpreted as no significant gap (category A), score of 21-28, minor to moderate gap/concern (category B) and score of 29-36, moderate to serious gaps/concern (category C).

The data for HIV was based on the United Nations (UNAIDS) estimation (THE WORLD BANK, 2018a; 2018b). Prevalence data for both gender (male and female) were obtained and analyzed using the Microsoft Excel Version 2010. Prevalence of HIV was defined as the percentage of the people with age 15-24 years who were infected with HIV. The average prevalence of HIV was obtained by getting the mean value from that of 2011 till 2017. The findings are presented in sentences, percentages and charts.

\section{RESULTS}

\section{Category of Curriculum}

Table 1 displays the category the curriculum of the 10 countries in East and Southern Africa that were studied falls into. Notably, none of the curriculum fell under the category of no significant gap. Therefore, all the curriculums have significant gap. The bulk of the countries $(60 \%)$ had minor to moderate concerns in their curriculum while the remaining $40 \%$ had moderate to serious gaps/ concerns.

Table 1. Category of CSE curriculum

\begin{tabular}{ll}
\hline Category B & Category C \\
\hline Botswana & Kenya \\
\hline Lesotho & Namibia \\
\hline Malawi & South Africa \\
\hline Swaziland (Eswatini) & Zambia \\
\hline Uganda & \\
\hline
\end{tabular}

Zimbabwe

Category B: Minor to moderate gap/concern in curriculum

Category C: Moderate to serious gap/concern in curriculum

\section{HIV Prevalence}

The trend of HIV prevalence among the young (15-24 years) in the ten African countries examined is presented in Figure 1 (males) and Figure 2 (females). The average HIV prevalence in these countries is displayed in Figure 3.

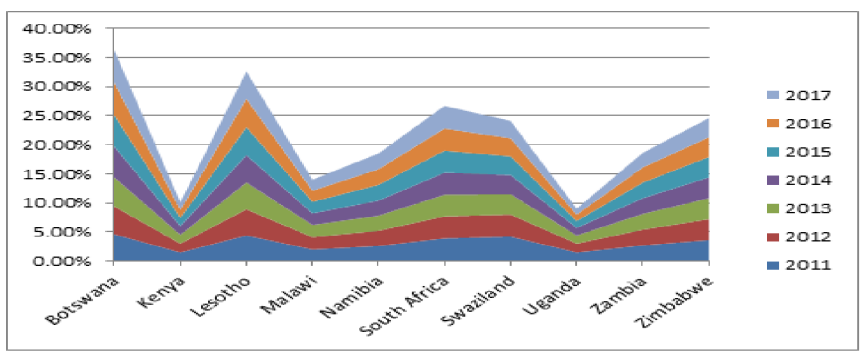

Figure 1. HIV prevalence among males within age 15-24 years 


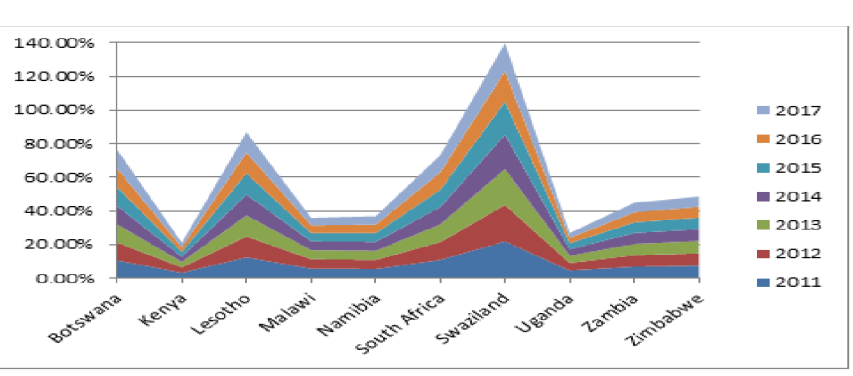

Figure 2. HIV prevalence among females age 15-24 years

This trend clearly shows that Botswana has the highest prevalence of HIV among young males (15-24 years). The countries with peaks are Botswana, Lesotho, South Africa and Zimbabwe. The trend of Kenya, Malawi and Uganda's HIV prevalence among young males form a valley. These countries have low HIV prevalence over years (2011-2017). Of all the countries examined, only Botswana had an increasing HIV prevalence from 2011 to 2017. Malawi, Namibia and South Africa's HIV prevalence among the young male population is almost the same been between 1.9-2.1\%, 2.6-2.7\%, 3.7-3.9\% respectively. However, Lesotho has been having mixture of declines and increase in HIV prevalence. It is of note that the HIV prevalence among young males in Kenya, Swaziland, Uganda, Zambia and Zimbabwe has been gradually decreasing since 2011.

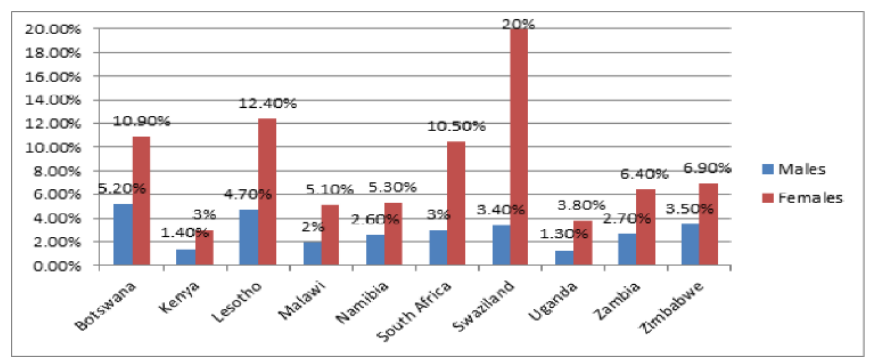

Figure 3. Average HIV prevalence among males and females within age $15-24$ years

The trend above shows that Botswana, Lesotho and Swaziland have high prevalence of HIV among young females while Kenya, Malawi, Namibia and Uganda have low prevalence. The prevalence of HIV among the young female population has been increasing from 2011. Botswana is the only country among the 10 studied that has a constantly increasing prevalence. Most of the countries (8) had declining prevalence of HIV prevalence from 2011. However, only Lesotho had a mixture of decline and increase in its HIV prevalence. The HIV prevalence declined from 2011 till 2015 after which it increased in 2017.

Swaziland had the highest average young female prevalence (20\%) of HIV among the countries studied while Kenya had the lowest (3\%). The country with the highest average prevalence of HIV among young males within age 1524 years in the 10 African countries examined is Botswana (5.20\%) and Uganda, the lowest (1.30\%). Therefore, the average prevalence of HIV among the young females range between $3-20 \%$ and males, $1.30-5.20 \%$. It is noteworthy that the prevalence of HIV among young females in Swaziland is about four times the prevalence of HIV among young males in Botswana which has the highest for the male population. The average HIV prevalence for all the countries for the male category is 2.78 and 7.56 for the female category.

\section{HIV Prevalence and Category of Curriculum}

Three of the six (50\%) countries with category B curriculum i.e. curriculum with minor to moderate gap or concern had high prevalence of HIV from 2011 till 2017 among young males within age 15-24 years. However, only one of the four (25\%) countries with category $\mathrm{C}$ curriculum i.e. curriculum with moderate to serious concern, had high prevalence of HIV from 2011 to 2017 among young males. Also, two of the countries with category B curriculum had low HIV prevalence among young males over the years been considered in this study. Only one country with category $\mathrm{C}$ curriculum had low prevalence of HIV from 2011 to 2017.

Findings from analysis of data on the HIV prevalence from 2011 to 2017 among young females within age 15-24 based on the category of curriculum showed that three of the six countries (50\%) with category B curriculum had high HIV prevalence and none among countries with category $\mathrm{C}$ curriculum had high HIV prevalence. Also, two of the six countries (33\%) with category B curriculum had low HIV prevalence and two of the four (50\%) countries with category C curriculum had low prevalence of HIV among the young females.

The average HIV prevalence among young males in countries with category B curriculum is 2.9 while that of countries with category $\mathrm{C}$ curriculum is 2.6 . Also, the average prevalence of HIV among the young females within age 15 to 24 years in countries with curriculum $B$ is 8.4 and that of the countries using category $C$ curriculum is 6.3. Only two of the countries under the category $\mathrm{B}$ curriculum have an average HIV prevalence value lower than the overall average value for males, while three countries of the four under the category $\mathrm{C}$ curriculum had an average HIV prevalence lower than the average for all countries. For the female group, 3 countries using category $\mathrm{C}$ curriculum had an average HIV prevalence below the average for all the countries been studied while three countries using category $\mathrm{C}$ curriculum had an HIV prevalence value higher than the total average value.

\section{DISCUSSION}

A good number of countries with comprehensive sexuality education curriculum that has minor to moderate gaps had high prevalence of HIV among males within age $15-24$ years. It would be expected that countries with better CSE curriculum would have lower prevalence of HIV (UNESCO and UNFPA, 2012), however this is not the case here and this finding can be attributed to a number of factors. One of these factors is the perception of some individuals that CSE fuels homosexuality. Some parents feel that CSE encourages early sexual debut, this is another major factor (domestic factor). These exist in Botswana, one of the countries with a relatively better CSE curriculum but still high HIV prevalence. Also in Botswana, it is reported that because CSE as a subject is not examinable therefore teachers and students do not take it serious. Culture 
and tradition (socio-cultural factor) makes it difficult for some teachers to speak about sexuality in the classroom as they believe it is not an appropriate discussion (UNESCO, 2017).

Lesotho is another country in this category with a relatively better CSE curriculum and yet high HIV prevalence. This finding can be attributed to inadequate financial resources to train teachers and inspectors in CSE. Also, there are inadequate human resources, especially teachers well trained to teach CSE (Mathabo, 2012). It is also reported that the teachers training institute did not prepare teachers adequately to teach comprehensive sexuality education (UNESCO, 2017).

None of the countries with CSE curriculum with moderate to serious gaps had high prevalence of HIV among young females (15-24 years). This finding can be because relatively more extensive works on implementing CSE has been done in these countries. Kenya is a country in this category and it is estimated that in Kenya, more than 2 million adolescents have been educated on CSE via digital platforms and also, more than 100 religious institutions have been properly educated on CSE. Additionally, as at 2014, more than 25,000 parents have been educated on CSE by the Centre for Adolescent Studies (UNESCO, 2017). Namibia also belongs to this category and there are full time teachers in place to implement CSE (UNESCO, 2017). South Africa, which is also in this category, has extended CSE to out-of-school youth (UNESCO, 2017). With all these in place in these countries, the reasons behind their lower prevalence of HIV can be easily understood.

In the male group, the average HIV prevalence between both categories (i.e. CSE category with minor to moderate gaps and the other with moderate to serious gaps) was very close with a difference of 0.3 . Although, countries with better CSE curriculum have higher HIV prevalence, a lot of organizations are working to tackle this challenge. This is responsible for the small difference in average HIV prevalence over years, from 2011 to 2015. In those countries with the relatively better CSE and high HIV prevalence, some success has been recorded. In Botswana for example, forty teachers training college teachers and some lecturers from the University of Botswana have been trained on the CSE pre-service curriculum. Through these training, more than 600 teachers have been trained in CSE. Also, an online course was delivered to 110 teachers from the 10 education regions and these teachers have been able to educate about 11,000 people (UNESCO, 2017). It is very possible that very soon, the difference in HIV prevalence in these two categories will be equal and that of countries with better comprehensive sexuality curriculum might even become lower than what exist in countries with poorer CSE curriculum.

This study found out that majority (4 out of 6 ) of the countries using CSE curriculum with minor to moderate gap had an average HIV prevalence among young males (15-24 years) higher than the average of all the 10 countries involved in the study. This finding can be attributed to a number of factors cross country across years. These factors include; culture and tradition, poor funding, inadequate training on teachers, lack of seriousness of students and teachers with CSE, inadequate number of teachers trained on comprehensive sexuality education, weak training of inspectors to monitor implementation of CSE in schools, misconceptions of some parents, elders, teachers and other individuals about comprehensive sexuality education, and many more (Pauchaud et al., 2018; UNESCO, 2017).

In the female category, one of the country using CSE curriculum with moderate to serious gaps had an average HIV prevalence higher than the average of all the 10 countries involved in the study. In this particular country (South Africa), this finding might be due to a number of reasons. One, CSE is taught for only two hours in a week. This is too short for all topics to be covered (UNESCO, 2017). Also, some teachers are reluctant to teach their students about comprehensive sexuality education because of their values, cultures and tradition. A very strong reason for the high prevalence of HIV among the young females in South Africa is the relatively weak CSE curriculum although these is been worked on (Badenhorst, 2018; UNESCO, 2017). The finding from South Africa seems to clearly show that poor CSE curriculum is related to high HIV prevalence.

\section{CONCLUSION}

The comprehensive sexuality education curriculum was developed to educate young minds about all components of sexuality namely emotional, cognitive, physical and emotional. The use of a curriculum following the guidelines for the standard CSE is expected to positively affect the young males and females in these countries. However, this is not the finding in this study. The higher prevalence of HIV in countries with better CSE curriculum may be due to poor implementation, culture and tradition, poor funding, among many others. It is therefore recommended that the factors responsible for these findings in those countries be addressed and this may help reduce the prevalence and burden of HIV.

\section{LIMITATIONS}

1. The curriculum review was carried out in 2011 and there might have been changes in the curriculum.

2. The presence of a curriculum does not necessarily translate to implementation of CSE curriculum. This might limit the accuracy of the findings of this study.

3. The HIV prevalence used was among young males and females within age 15-24 years. This might limit accuracy of the findings of this study as most of the school aged children are within age 8-20 years.

\section{RECOMMENDATIONS}

1. A review should be carried out to assess the CSE curriculum in all African countries in line with the standard CSE curriculum. The latest one available is for only ten countries in Africa and it was carried out 8 years ago.

2. An African wide study should be carried out to investigate the implementation of CSE curriculum. The findings from such study would be helpful in finding connections between CSE implantation and 
various variables and indicators of significance or interest.

3. The number of hours assigned to teach CSE should be increased. The number of hours used presently is too little and not enough to cover the curriculum.

\section{REFERENCES}

Badenhorst, N. (2018). CSE in South African Schools Sexuality Education, or Sex Education? Available at: https://forsa.org.za/cse-in-south-african-schoolssexuality-education-or-sex-education/

Keogh, S. C., Stillman, M., Awusabo-Asare, K., Sidze, E., Monzón, A. S., Motta, A. and Leong, E. (2018). Challenges to implementing national comprehensive sexuality education curricula in low- and middle-income countries: Case studies of Ghana, Kenya, Peru and Guatemala. PLoS ONE 13(7), e0200513. https://doi.org/10.1371/ journal.pone.0200513

Kidd, C. and Hayden, B.Y. (2015). The psychology and neuroscience of curiosity. Neuron, 88(3), 449-460. https://doi.org/10.1016/j.neuron.2015.09.010

Kipping, R. R., Campbell, R. M., MacArthur, G. J., Gunnell, D. J. and Hickman, M. (2012). Multiple risk behaviour in adolescence. $J$ Public Health, 34(1), i1-i12. https://doi.org/10.1093/pubmed/fdr122

Kohi, A., Remy, M. M., Binkurhorhwa, A. K., Mitima, C. M., Mirindi, A. B., Mwinja, N. B., Banyewesize, J. H, Ntakwinja, G. M., Perrin, N. A. and Glass, N. (2018). Preventing risky behaviours among young adolescents in eastern Democratic Republic of Congo: A qualitative study. Glob Public Health, 13(9), 1241-1253. https://doi.org/10.1080/ 17441692.2017.1317009
Kyilleh, J. M., Tabong, P. T. and Konlaan, B. B. (2018). Adolescents' reproductive health knowledge, choices and factors affecting reproductive health choices: a qualitative study in the West Gonja District in Northern region, Ghana. BMC Intl Health Hum Rights, 18(6), 1-12. https://doi.org/ 10.1186/s12914-018-0147-5

Mathabo, K. (2012). Sexuality education in rural Lesotho schools: challenges and possibilities. Sex Education, 12, 411-423. https://doi.org/10.1080/14681811.2012.677210

Panchaud, C., Keogh, S. C., Stillmah, M., Awusabo-Asare, K., Motta, A., Sidze, E. and Monzon, A. S. (2018). Towards comprehensive sexuality education: a comparative analysis of the policy environment surrounding schoolbased sexuality education in Ghana, Peru, Kenya and Guatemala. Sex Education, (3), 277-296. https://doi.org/ $10.1080 / 14681811.2018 .1533460$

SIMBIHealth C2019). Sexuality Information, Mental and Behavioural Initiative for Health Project. Available at: https://www.srhin.org/causes.php

The World Bank. (2018a). Prevalence of HIV, female (\%ages 1524). Available at: https://data.worldbank.org/indicator/ SH.HIV.1524.FE.ZS?view=chart

The World Bank. (2018b). Prevalence of HIV, Male (\%ages 1524). Available at: https://data.worldbank.org/indicator/ SH.HIV.1524.FE.ZS?view=chart

UNESCO and UNFPA. (2012). International technical guidance on sexuality education. Available at: https:/www.unaids .org/sites/default/files/media_asset/ITGSE_en.pdf

UNESCO. (2017). CSE Scale up in Practice: Case studies from eastern and southern Africa. Available at: https://hivhealthclearinghouse.unesco.org/sites/default/ files/resources/cse_scale_up_in_practice_june_2017_final_ .pdf

UNFPA. (2016). Comprehensive Sexuality Education. Available at: www.unfpa.org/comprehensive-sexuality-education 